\title{
Prenatal Development and Micro-Vasculatures of the Eyelids of the Egyptian Water Buffalo (Bubalus buba- lis)
}

\author{
Ahmed Sayed-Ahmed \\ Department of Anatomy and Embryology, Faculty of Veterinary Medicine, Alexandria \\ University, Damanhur branch, Bostan, Egypt.
}

With 5 figures

Received August 2009, accepted for publication November 2009

\begin{abstract}
Twenty-six buffalo fetuses ranging from 6.2- $103 \mathrm{~cm}$ CVRL (56 - 305 days-old) were used in this study. Twenty fetuses were used to study the eyelids morphogenesis and six fetuses were used to study the vasculogenesis and microvasculatures of the eyelids. The fused superior and inferior eyelids were recognized at $6.2 \mathrm{~cm}$ (56 days). The fusion started to separate at $50 \mathrm{~cm}$ CVRL (186 day) while the eyelids appeared grossly opened at $60 \mathrm{~cm}$ CVRL (209 days). The palpebral skin and conjunctiva exhibit remarkable developmental changes throughout the gestation period. The primordia of the hair follicles and orbicularis oculi muscle were observed at 10.5 $\mathrm{cm}$ CVRL (76 days). The primordium of the tarsal glands was seen at $13 \mathrm{~cm}$ CVRL (87 days), while the primordia of the sweat and sebaceous glands were first demonstrated in fetus of $21 \mathrm{~cm}$ CVRL (121 days). Keratinization of the palpebral skin started at $38 \mathrm{~cm}$ CVRL (159 days) while the cornified skin with complete cutaneous structures and the papillae of tarsal glands openings were seen at $60 \mathrm{~cm}$ CVRL (209 days), thus it might be associated with eyelids reopening. The microvasculature of the eyelids was started to differentiate into vascular net-works at $19 \mathrm{~cm}$ CVRL (114 days), while the three cutaneous vascu-
\end{abstract}

lar networks, the perifollicular vascular networks of the cilia besides the mucosal and sub-mucosal networks of the conjunctiva appeared fully developed at $80 \mathrm{~cm}$ CVRL (254 day). Finally one could conclude that the early differentiation and maturation of eyelids microvasculature might be associated with the early development of the eyelids structures.

\section{Key words}

Morphogenesis, eye lids, Buffalo

\section{Introduction}

The development of the eyelids requires coordinated cellular proce-sses of proliferation, cell shape changes, migration and cell death (Tao et al., 2005). Many congenital and developmental abnormalities of the mammalian eye have been (Zenz et al. 2003). These abnormalities are probably the results of either hereditary aberration of growth or may result from exogenous influences, such as teratogenic substances or infectious diseases (Kahrs et al. 1970; Zenz et al. 2003; and Zhang et al., 2003).

The eyelids protect the eye from mechanical injuries and dazzling light. Repeating automatic closure of the lids 
(blinking) helps in the distribution of lacrimal fluid and has a pumping effect on the lacrimal sac. The tissue layers of the lids from the front to back are as follow: skin, subcutaneous, striated skeletal muscle (palpebral part of the orbicularis oculi), submuscular areolar tissue, tarsal plates, nonstriated muscle, and conjunctiva (Csillag, 2005). Eyelid development, closure and subsequent reopening are a feature common to all mammals. Whether a particular animal species is born with its eyelids open or closed is believed to be deter-mined by the stage of development of the animal at the time of birth (Findlater et al., 1993). The developmental steps of the eyelid have been described in several species such as bovine embryo (Bister et al., 1973) dog embryo (Aguirre et al., 1972), one humped camel (Zayed, 2004) and human embryo (Hamilton and Mossman, 1972, and Pearson, 1980). The palpebral microvascularization in human is dependent on arterial arcades fed by two sources: a lateral arterial source composed of the lateral palpebral arteries and medial arterial source, formed by the medial palpebral arteries. The anastomosis between the lateral and medial palpebral arteries creates an arcade at each eyelid (Tucker and Linberg, 1994). In human fetuses and neonates the microvasculatures of the superficial part of eyelid are split into papillary, superficial reticular and deep reticular networks, while the micro-vasculatures of the palpebral conjunctiva are split into mucosal and submucosal networks (Wolfram-Gabel and Sick, 2002). So far there is no available information deal with prenatal development of the eyelids of the Egyptian water buffalo and there is no information about the vasculogenesis and microvasculatures of the eyelids of the domestic animal. Thus the aim of the present study is to look into the morphogenesis, vasculogenesis and micro- vasculatures of the eyelid of the Egyptian water buffalo.

\section{Materials and Methods}

This study was carried out on $26 \mathrm{em}-$ bryos and fetuses of the Egyptian buffaloes of both sexes and ranging from 6.2 $103 \mathrm{~cm}$ CVRL (56 - 305 days-old). These embryos and fetuses were collected from Tala and Tanta slaughter houses, under emergency slaughter, and from the aborted and the premature-birth fetuses on the veterinary field by personal communication with the veterinarians. For morphogenesis, the embryos and fetuses of $6.2-15 \mathrm{~cm}$ CVRL (56- 96 days-old), were fixed in $10 \%$ neutral buffered formalin. While upper and lower eyelids of the older fetuses were excised and were fixed in $10 \%$ neutral buffered formalin. The whole eyes from 6.2 - $15 \mathrm{~cm}$ fetuses and the specimens of upper and lower eyelids of older fetuses were processed with the normal histological technique of dehydration, clearing and paraffin embedding. sagittal sections $5-7 \mu$ thick, were cut on Leitz rotatory microtome and stained with hematoxylene and eosin, trichrome, and PAS stains adopted by Drury and Wallington (1980). In the same time the eyelids were examined by stereomicroscope.

For vasculogenesis and microvasculatures of the eyelid, the fetuses of 19 , 23, 38 46, 65 and $80 \mathrm{~cm} \mathrm{CVRL} \mathrm{were}$ perfused with a warm normal saline solution, and then injected with an equal mixture of Indian ink and bovine serum (1:1) through the umbilical arteries and / or the umbilical veins. The injected fetuses were fixed in $10 \%$ neutral formalin buffered solution for 1-2 weeks. The removed eyelids were dehydrated in ascending grades of alcohol and cleared in benzol followed by an equal mixture of methyl benzoate and 
benzyl benzoate according to (Fath ElBab et al., 1983). The prepared eyelids were examined by the aid of light and binocular dissecting stereomicroscopes, however the clearing method mentioned by Fath El-Bab et al., (1983) was limited to young age fetuses due to the thick complex structures of the eyelids. Therefore in this study I adapted new method based on (Wolfram-Gabel and Sick, 2002) where the Indian ink perfused upper and lower eye-lids were removed dehydrated by ascending grades of ethanol, cleared with xylol and paraffin embedded. The blocks were cut in a sagittal or frontal plane into a series of $(250 \mu \mathrm{m})$ slices. Then these thick slices were trans-parified and cleared in benzol followed by an equal mixture of methyl benzoate and benzyl benzoate. The prepared eyelids slices were examined by the aid of the light and binocular dissecting stereomicroscopes. In the same time thin sections $(5-10 \mu \mathrm{m})$ were cut and stained with hematoxylene and eosin stain. The nomenclature used in this work was adapted to Wolfram-Gabel and Sick (2002) and Nomina Anatomica Veterinaria (2005) whenever, it was possible. The ages of the collected buffalo embryos and fetuses were estimated by the formula given by Abd El-Raouf and El-Naggar (1968).

\section{Results}

The eyelids buds of the buffalo were developed early before $6.2 \mathrm{~cm}$ CVRL (56 days) where the fusion of both buds was recognized at this age in front of the eyeball. By stereo and light microscope, the fusion started to separate at $50 \mathrm{~cm}$ CVRL (186 days) while the complete separation of the upper and lower eye-lids was grossly observed at $60 \mathrm{~cm}$ CVRL (209 days). This separation was associated with appearance of both eyelid cilia and the small papillae of tar- sal glands openings. The cilia of both upper and lower eyelids were directed rostrally and ventrally and were observed to be the second hair appeared in buffalo after the eyebrow (Fig. 1 A-D). Based on the main developmental events that were observed, the entire development of the eyelids can be divided into the five stages.

First stage (6.2 - $8.5 \mathrm{~cm}$ CVRL 56- 67 days old): early on this stage the upper and lower eyelids of the buffalo were formed of upper and lower ectodermal folds with mesenchymal cores in front of the eyeball. The two folds were completely fused at this age making a closed conjunctival sac that bounded posteriorlly by the developing cornea and anteriorly by the fused developing upper and lower eyelids. The fusion was formed of an aggregation from the superficial layers of the developing epidermis that appears as a mass of pale polyhedral cells with centrally located nuclei (Fig. 1E). The polyhedral cell mass at the fusion was flanked superiorly and inferiorly by a well defined basal cell layer that was columnar with an elongated nucleus. The superficial polyhedral cells of the fusion site formed of several layers on the adjacent epidermal and conjunctival surfaces. A distance from the site of the fusion of the epidermal surface of the eyelids was covered with basal cell layer and outer 2-3 layers of polyhedral cells, while the conjunctival surface was covered with only basal cell layer. The mesenchymal core of each developing eyelid was formed of loosely arranged polyhedral or spindle shaped cells with vesicular nuclei. Small blood spaces with blood cells were observed in the dermis. In late period of this stage, the superficial cells of the epidermis were 34 layers while in conjunctival surface an additional single polyhedral cell layer was added to the basal cells layer. The 
third eyelid appeared as outgrowth from the conjunctival surface of the developing upper eyelid and extended distally within the closed conjunctival sac between the eyelid and cornea. The first structure of the third eyelid was similar to the upper and lower ones where it was formed of anterior and posterior basal cell layer inclosing a mesenchymal core and covered by superficial pale polyhedral cells. (Fig.1 F).

\section{Second stage (10.5-15 cm CVRL 76-}

96 days): early on this age the epidermis increased in thickness with 5-6 layers of superficial cells that become 6-8 in late period while conjunctival surface was covered with only two cell layers. The primordia of both orbicularis oculi muscle and hair follicle were developed. The orbicularis oculi muscle was observed within the eyelid between the epidermis and palpebral conjunctiva as eosinophilic muscle cells while the hair follicle was observed as local proliferation and condensation of epidermal basal cells forming a hair bud or plug (Fig $2 \mathrm{~A}$ and $\mathrm{B}$ ). In mid period of this stage (13 cm CVRL 87 days), the hair bud became thickened and deeply invaginated within the dermis. In this period, the primordia of the tarsal gland appeared as inward growth of epidermal basal cells at the junctional (fusion) zone of the eyelids (Fig. $2 \mathrm{C}$ ).

In late period of this stage, the hair follicles showed different stages of development where the older one at the site of the developing cilia was circular cellular mass with outer dark nucleated cuboidal cell layer and centrally located several pale polyhedral cells. In a sagittal view, the hair follicle appeared as solid cords of cells where its peripheral cells were similar and continuous with the basal cells of the epidermis. Newly developed hair follicles appeared especially a distance from the junctional zone. The tarsal glands enlarged and deepened within free edges of the eyelid in the form of a pocket like invagination (Fig 2D). The primitive blood spaces (island) become numerous at the dermis and the subconjunctival area. The outer cells of the primitive blood islands were differentiated and gave rise to the flattened endothelial cells of the primitive blood vessels, while, the centrally located cells were differentiated into the primitive blood cells. (Fig 2D)

Third stage (19 - $23 \mathrm{~cm}$ CVRL, 114126 days): The hair follicles increased in length and extended more deeply within the dermis with constricted neck and broad shaft. In mid period of this $(21 \mathrm{~cm})$ the sweat glands began to build up as small solid cellular swellings that were originated from the upper third of the hair follicles. Also the primordia of the sebaceous glands appeared as small cellular budding from the lower third of the growing follicles (Fig. $2 \mathrm{E}$ ). The tarsal gland extended more deeply within the eyelids and began to give several outgrowth branches; firstly the branches were formed of solid cord of single cuboidal cells layer while the more advanced branches were formed of outer wall of cuboidal cells with centrally located pale polyhedral cells. These outgrowths represented the developing acini and its connecting ductules. The main lumen of the developing tarsal glands was representing the main duct of the tarsal glands, till this stage the lumen was filled by several layers of pale polyhedral cells that were freely communicated with the pale polyhedral cells of the junctional zone (Fig. 2 E). In late period the hair follicles showed further development where the solid follicles were developed into tubular structure with the peripheral cells similar and connected to the basal cells of the epidermis and central pale polyhedral cells that were similar and connected to the 
superficial cells of the epidermis. Orbicularis oculi muscle was more developed and started to arrange into the muscle bundles (Fig. $3 \mathrm{~A}$ and $\mathrm{B}$ ).

During this stage the vascula-tures of the eyelid started to differentiate into vascular net-works or plexuses that were originated from large deeply located palpebral vessels. From the latter vessels the cutaneous vessels pass anterioraly toward the deep part of the dermis and then became nearly parallel to the epidermis. Within the deep part of the dermis the cutaneous vessels gave rise to several collateral branches to form the deep reticular network. At the conjunctival part of the eyelid a large vessel was observed passing nearly parallel to conjunctiva (Fig. $2 \mathrm{~F}$ and $\mathrm{G}$ ). The large palpebral artery passed parallel to the palpebral fissures and it represented the anastomosis between the lateral and medial palpebral arteries. Along its length it gave off several branches toward the palpebral fissures. Large veins pass toward the fissures where it divided forming venous loops that gave off several satellite branches for the arterial side toward the palpebral fissures (Fig. $3 \mathrm{C}-\mathrm{E}$ ).

Fourth stage (38- $50 \mathrm{~cm}$ CVRL, 159 186 days): early in this stage the epidermis was formed of 8-9 cell layers with basement membrane between epidermis and dermis and toward the surface the superficial cells of the epidermis became flattened with the first evidence of keratinization. The hair follicles became more differentiated and the upper part of the hair canal of the early developed hair appeared clear and protruded through the epidermis, while a distance from the eyelid margin there were few newly developed hair follicles. The sweat glands increased in length and deepened toward the dermis and were composed of two layers of cuboidal cells. Sebaceous glands were more differentiated and the early developed one appeared as semi-lobulated glands with several acini around the distal half of the hair follicles. (Fig. $3 \mathrm{~F}$ ). In late period of this stage, signs of eyelids separation were observed where the cell layers of fusion zone were split into dorsal and ventral masses each of it remained attached to the free margin of its corresponding eyelid. The epidermis was more differentiated with appearance of the melanin pigment especially at the free margin of the eyelid. The keratinized hair cores started to occupy the entire thickness of the epidermis. The conjunctiva appeared thicker especially toward the eyelid margin and formed of 4-5 layers of stratified squamous epithelium while a distance from the eyelid margin was formed of 2-3 layers of only flat cells. The duct of tarsal glands had a patent lumen with a stratified squamous epithelium (Fig. $3 \mathrm{G}$ ). The vasculatures of the eyelid become more abundant, the deep reticular net-work gave off several branches toward the epidermis to form the superficial reticular network. At the conjunctiva the large main vessels detached several vessels that pass perpendicular to the conjunctiva forming the sub-mucosal network of the conjunctiva (Fig. $4 \mathrm{~A}$ and $\mathrm{B}$ )

Fifth stage could be divided into two developmental periods. The first period (60-80 cm CVRL, 209-254 days), the whole strata of the keratinized epidermis were fully differentiated and developed into the stratum basale, stratum spinosum and stratum corneum. The melanin pigments were progressively increased from the beginning to the middle of this period then markedly reduced at its end. The epidermis decrease in thikness (from 70 to $50 \mu \mathrm{m}$ ), while the dermis became thick and the dermal ridges (papillae) were developed and interdigitate with invaginations of 
the epidermis (epidermal ridges). The keratinized hair shaft extended through the previously developed hair canal. Few tactile hairs can be recognized a distance from the eyelid margin. During this period the openings of the tarsal glands were the landmark between the keratinized skin and non keratinized palpebral conjunctival side of the eyelid. The conjunctiva was formed of stratified squamous epithelium (Fig $4 \mathrm{C}$ )

In the second period (92- $103 \mathrm{~cm}$ CVRL (281- 305 days), the thickness of epidermis at the free edge between the cilia and openings of the tarsal glands was about $100 \mu \mathrm{m}$ while a distance from the free edges the epidermis was thinner and measured about $45 \mu \mathrm{m}$. The dermal papilla at the free edge was about $25 \mu \mathrm{m}$ and a distance from the margin was about $10 \mu \mathrm{m}$. The cilia were fully developed and more numerous in the upper eyelid than that in the lower eyelid and disappeared toward the commisures. The tactile hair appeared larger than normal hair and was surrounded with large blood sinuses and thick collagenous capsules. The sebaceous gland became enlarged lobulated and was separated from the tarsal glands by bundles of the orbicularis muscles. The sweat glands appeared deeply situated and lined with simple columnar epithelium (Fig. $4 \mathrm{D}$ and E). The tarsal glands and its openings were fully developed and were more numerous in the upper eyelid (32 glands) than in the lower eyelid (25 glands). The glands were divided into several lobules that were connected by a short duct into the main duct of the glands. Both of the lobules ducts and the main duct of the gland were lined by stratified epithelium while at the opening of the gland the main duct was lined by keratinized squamous epithelium. The gland was surrounded by a thick collagenous tarsal plate (Fig. $4 \mathrm{~F}$ and $5 \mathrm{~A}$ ). Just post- erior to the openings of the tarsal glands, the conjunctival epithelium was stratified squamous non-keratinized with few melanin pigments, then it changed into transitional epithelium with goblet cells which increased toward the fornix (Fig. 5 B).

In $80 \mathrm{~cm}$ CVRL (254 days), the vasculature of the eyelid became more abundant, the superficial vascular reticular network was composed of vessels with abundant anastomoses, creating a flattened network, parallel to the epidermis. From the latter networks small capillary loops passed and occupied the dermal papillae of the dermis forming the vascular papillary networks. The submucosal network of the conjunctiva became more intensive and detached several small vessels that pass toward the conjunctiva forming the mucosal network which was parallel to the surface and formed a polygonal mesh of small vessels (Fig $5 \mathrm{C}-\mathrm{E}$ ). In this age there were marked formations of intensive perifollicular vascular networks around the hair follicles (Fig. $5 \mathrm{~F}$ ).

\section{Discussion}

According to the available literatures, this study reported the first information about the development of the eyelids of the Egyptian water buffalo and the first information about the vasculogenesis and micro-vasculatures of the eyelids of a domestic animal. Eyelids development and closure was established in the buffalo at $6.2 \mathrm{~cm}$ CVRL (56 days) while the subsequent reopening was observed at $50 \mathrm{~cm}$ CVRL (186 days). These develop-mental steps are a feature common to all mammals with these timetable differences from species to another. In bovine embryo the lid buds appear and fuse at $1.5 \mathrm{~cm}$ and $4 \mathrm{~cm}$ CVRL respectively, but reopen at birth (Bister et al., 1973). In the one humped 
camel, the lid buds appear, fuse and reopen at $2.4 \mathrm{~cm}, 8.4 \mathrm{~cm}$ and $66 \mathrm{~cm}$ CVRL respectively, (Zayed 2004). In the dog embryo the lid buds appear at gestational age 28 days fuse at 32-33 days of gestation and reopen at 14 days after birth (Aguirre et al., 1972). In human embryos, the eyelids start to form about 40-45 days of prenatal life with complete fusion occurring approximately 15 days later (Pearson, 1980). The eyelids remain closed thereafter until the seventh month of the fetal life when they reopen (Hamilton \& Mossman, 1972). A particular animal species is born with its eyelids open or closed is believed to be determined by the stage of development of the animal at the time of birth (Findlater et al., 1993).

Sevel (1988) in human, divided eyelid development into 5 stages: (1) development of eyelid folds, (2) stage of eyelid fusion, (3) development of specialized features, (4) eyelid separation and (5) eyelid maturation. Nearly the same stages of eyelid development were observed in buffalo although the timing of events is different. However, bearing in mind this time difference, there is a close similarity in the relative duration of each stage of development. The shortest stage in buffalos, bovine (Bister et al., 1973), camel (Zayed 2004), dog (Aguirre et al., 1972), human (Pearson, 1980) and mouse (Findlater et al., 1993) was that of eyelid fold development and fusion. The longest stage of these species was from the time of eyelid fusion through to the time when the eyelids finally separate. It is during this latter stage, the specialized structures of the eyelids are developed.

The skin covering of buffalo eyelid showed prenatal changes. During first, second and third stages the skin covering increased in thickness due to addition of more cell layers. During the fourth stage, the skin covered still in- creased in thickness due to addition of more layers as well as the beginning of keratinization that associated with eyelids separation. The association of keratinization and eyelids separation was recorded by Williams and warwick (1980) in human and Teraishi and Yoshioka (2001) in mouse.

During the early period of the $5^{\text {th }}$ stage, the skin cover increased in thickness while at late period and in full term fetuses the skin covering was markedly thinner. These pattern of developmental changes were in agreement with ElSkhawy (1973) in the skin of shoulder region, trunk and thigh regions of buffalo fetus and with (Zayed, 2004) in the eyelid of camel. In the present study, the epidermis of 281-305 days old fetus was about $45 \mu \mathrm{m}$, while in adult buffalo it measure about $63 \mu \mathrm{m}$ (Hifny et al., 1985). El-Skhawy (1973) mentioned that the epidermis of shoulder region, trunk region and thigh region of 10 month buffalo fetus measures $77.6 \mu \mathrm{m}$. The thinning of the epidermis of the eyelid appeared to be logic pre-requisite to an easy movement of the eyelids.

In the present study, the first evidence of both orbicularis oculi muscles and hair follicles of the eyelids were noticed at $10.5 \mathrm{~cm}$ CVRL (76 day). Association of orbicularis oculi muscle and hair follicles development was also reported in $9 \mathrm{~cm}$ CVRL bovine fetuses (Bister et al., 1973) and dog by gestational day 40 (Aguirre et. al., 1972). This association was not reported in camel where the first indication of the hair follicles and orbicularis oculi muscle was seen at $8.4 \mathrm{~cm}$ and $19 \mathrm{~cm} \mathrm{CVRL}$, respectively (Zayed, 2004). The appearance of new hair follicles of buffalo fetuses lasts until $38 \mathrm{~cm}$ CVRL (159 days) and the cilia (eyelashes) began to emerge at 50 cm CVRL (186 days). El-Skhawy (1973) recorded that, the hair follicles of shoulder, trunk and thigh regions start to ap- 
pear at the end of 3rd month and last to the end of the 4th month. and the hair emerged at the 7th month. During this study a few emerged hairs were grossly observed by naked eye on the eyebrow at $29 \mathrm{~cm}$ CVRL (139), thus one could conclude that the eyelids may be the second place in the body of the buffalo in which the initiation of the hair follicles occur. Zayed (2004) in camel, concluded that the eyelids are the first place in the body because he noticed the initiation of the eyelid hair follicles of camel fetuses at $8.4 \mathrm{~cm} \mathrm{CVRL}$ and the new follicles last until $41 \mathrm{~cm}$ CVRL. , Duogbag and berg (1983a) reported the first hair initiation in the prescapular region of $15 \mathrm{~cm}$ CVRL camel fetuses and the addition of new follicles lasts until $50 \mathrm{~cm}$ CVRL.

The present study revealed the development of the sweat and sebaceous glands at the $21 \mathrm{~cm}$ CVRL (121 day). This result is agreed with the report of (El-Skhawy, 1973) in the shoulder, trunk and thigh regions of buffalo fetuses for the sweat gland only. The same author reported the development of the sebaceous gland at 4.5 month old buffalo fetus. Zayed (2004) reported the development of both sweat and sebaceous glands in the eyelids of camel at 36.5 $\mathrm{cm}$ CVRL, while Duogbag and berg (1983b) in camel fetuses reported the same onset for the sweat glands, however they noticed the sebaceous glands at a latter stage (45 cm CVRL).

In the present study, the tarsal glands appeared for the first time at $13 \mathrm{~cm}$ CVRL (85 days) as large sebaceous like gland not associated with the hair follicle during any stages of its development. This absence of association is agreed with Doxanas and Anderson (1984) in human and Diesem (1975) in ruminant. Although the development of the eye and eyelid was reported in some domestic animal, none of the lite-

J. Vet. Anat. ratures paid an attention to the development of tarsal glands. In mouse the gland appeared early by gestational day 15 (Findlater et al., 1993). The tarsal gland histology and ultrastructures were described in the buffalo (Prasad and Sinha, 1979; Hifny et al., 1985) in steer, human, primates and rabbits (Jester et al., 1981). In this finding and based on the openinigs of tarsal glands the numbers of the glands were 32 and 25 in the upper and lower eyelid, respectively. Diesem (1975) in bovine stated 35 glands in the upper lid and 25 in the lower lid. Whereas Hifny et al. (1985) in buffalo described that the tarsal glands are large and more numerous in the superior than in the inferior eyelid without mention of the numbers. The glands are multi-lobular with a large central duct lined with stratified squamous epithelium and at its opening acquired stratum corneum. This result is in consistence with Hifny et al., (1985) in buffalo and Jester et al., (1981) in steer. The duct of the tarsal glands had patent lumen at $50 \mathrm{~cm}$ CVRL age of eyelids separation. So tarsal glands maturation might be related to eyelid separation. This speculation is agreed with the statement of Doxanas and Anderson (1984) in human where they reported that, the secretion of these glands may facilitate the separation of the fused eyelids during the sixth month of gestation. The tarsal glands are absent in camel (Ibrahim, 1990) and (Zayed, 2004). In near and full-term fetuses the palpebral conjunctiva has tratified squamous epithelium non-cornified at the free border of the eyelids then changed into transitional epithelium with goblet cells. These observations agree with Hifny et al. (1985) in adult buffalo. The presence of transitional epithelium is an adaptation with the conjunctiva which is subjected to stretching and relaxation during eyelids movements. The goblet cells with its mucous secre- 
tion minimize the friction between the palpebral and bulbar parts of the conjunctiva.

In 56 days-old buffalo fetuses the blood vessels of the eyelids are represented by irregular islets of primitive blood cellular elements and at 94 days old by thin walled blood capillary vessels lined with an interrupted endothelium. These findings simulates that described for the cutaneous vessels in buffalo (El-Morsy, 1997) and El Ashry (2005), in 50-80 mm CVRL swine fetuses, Ahmed et al., (1984) and Zammaretti and Zisch (2005) in different animal species. The cutaneous vascular networks (plexuses) of the eyelids started to develop at 19 cm CVRL (114 day). While the three cutaneous networks besides the mucosal and submucosal networks of conjunctiva became fully developed at 80 cm CVRL (254 day). The presence of three cutaneous vascular networks in the eyelids and two vascular networks supplying conjunctiva agree with the statement of Wolfram-Gabel and Sick (2002) in human eyelid. Schummer et al. (1981) and Dyce et al. (2002) in different animal species reported three arterial and venous plexuses at superficial, middle and deep dermal levels. On the other hand Fath El-Bab et al., (1983) in bovine, El-Morsy (1997) and El Ashry (2005) in buffalo reported nearly the same architecture of skin vasculatures but with different nomenclatures, where they stated the presence of Rete arteriosum dermale, Rete arteriosum subpapillare organized and oriented at the deep and middle dermal layer respectively and subepidermal net-work which provides the nutrition for the developing epidermis. They also stated the presence of three venous plexuses, Plexus venosus sub-papillaris superficialis, Plexus venosus subpapillaris profundus and Plexus venosus dermis profundus.
In present study the mature constructive pattern of the cutaneous vascular architecture of the eyelid is observed at 80 cm CVRL (254 days). While the equivalent mature pattern of buffalos skin are observed in 289 and 283 days old fetus (El-Morsy, 1997) and (El Ashry, 2005) respect-tively, and in 8 month bovine fetuses (Fath El-Bab et al., 1983). The complete perifollicular vascular networks were fully developed in the eyelids at $80 \mathrm{~cm}$ CVRL (254 day). While in other skin regions the perifollicular vascular networks developed in 261-289 days-old buffalo fetuses (El-Morsy, 1997), in 283 days-old buffalo fetuses (El Ashry, 2005) and in 8-10 month bovine fetuses Fath El-Bab et al. (1983). Finally one could conclude that the early vasculogenesis of the eyelids is associated with the early development of the eyelid structures.

\section{References}

Abd El-Raouf, M. and El-Naggar, M. A. (1968): Biometery of the Egyptian buffalo fetus. J. Vet. Sci; U. A. R. 5: 37-43.

Aguirre, G., Rubin, L. and S.I. Bister, S I. (1972): Development of the canine eye. Am. J.Vet. Res 33: 2399-2424.

Ahmed, M. A. A.; Fath El Bab, M. R. R.; Schwarz, R. and Godynicki, S. (1984): The morphogenesis of the vasculature in swine fetal skin. Gegenbaurs morph. Jahrb; Leipzig 130: 297-306.

Bister, S. I., Rubin, L. and Aguirre, G. (1973): Development of the bovine eye. Am. J. Vet. Res 34: 712

Csillage, A. (2005): Atlas of the sensory organs functional and clinical anatomy. Humana Press Totowa, New Jersey pp 85-164. 
Diesem, C. (1975): The organ of vision (chapter 36 ruminant sense organs and common integument) in Getty, R.: Sisson and Grossmans the anatomy of the domestic animals 5th edition volume 1. pp 1180-1204. W.B Saunders Company. Philadelphia. London. Toronto.

Doxanas, M.T., and Anderson R.L. (1984): Embryology of the eyelids, lacrimal system and orbit. In: Doxanas MT, Anderson RL, eds. Clinical Orbital Anatomy. Baltimore, Md: Williams and Wilkins; pp 1-18.

Drury, R. A. B., and Wallington, F. A. (1980): Carlton's histological techniques, 5th Ed. Oxford University Press, Oxford, Toronto, New York.

Duogbag A.S.A.M.; and Berg, R. (1983a): The prenatal development of hair follicles and hair in the one-humped camel (Camelus dromedaries). Z Mikrosk. anat. Forsch. Leibezig 97: 886893.

Duogbag A.S.A.M. and Berg, R. (1983b): The development of the sweat and sebaceous glands in the skin of the onehumped camel (Camelus dromedaries). Z Mikrosk. anat. Forsch. Leibezig 97: 894-902

Dyce, K. M.; Sack, W. O. and Wensing, C. J. (2002): The Common integument, Chapt 10, page 347. In: Text Book of Veterinary Anatomy, 3rd Ed. Philadelphia, and London

El Ashry, M. (2005): cutaneous angiogenesis of the buffaloes (Bubalus bubalis). Thesis $M$. Vet. Sci. Faculty of Veterinary Medicine, Mansoura University.

El-Morsy, S. El-M. (1997):
Cutaneous angiogenesis in the Egyptian buffaloes (Bos bubalis L). Zag. Vet. J. 25: 67-82.

El-Sakhawy, M. A. (1973): The prenatal development of skin and hair in Egyptian buffaloes. Thesis, M. Vet .Sci. Fac. Vet. Med. Cairo Uni. Egypt

Fath El- Bab, M.R.; Schwarz, R. and Godynicki, S. (1983): The morphogenesis of the vasculature in bovine fetal skin. J. Anat. 136: 561-572.

Findlater, G.S.; Mcdougall, R.D. and Kaufman, M.H. (1993): Eyelid development, fusion and subsequent reopening in the Mouse. J. Anat. 183: 121-129,

Hamilton W.J. and H.W Mossman (1972): Growth of the embryo and fetus; development of external form; estimation of embryonic and fetal age. In Human Embryology, pp. 174-191. Cambridge: W. Heffer

Hifny, A.; Hassan, A.H.S. Selim, A. A.A. and Moustafa, M.N. (1985): Histological and histochemical studies on the eyelids of the buffalo in Egypt. Assiut Vet. Med. J. 14: 29-39

Ibrahim, M.T. (1990): surgical anatomical study on the appendages of the eye in camel, buffalo and donkey. Ph.D Thesis, Assiut University.

Jester, J.V. Nicolaides, N., Ronald E. and Smith, R.E. (1981): Meibomian gland studies: histologic and ultrastructural investigations. Invest. Ophthalmol. Vis. Sci. 20: 537-547.

Kahrs, R.F., Scott, F.W. and deLahunta, A. (1970):

Congenital cerebellar hypoplasia and ocular defects in calves following bovine viral diarrhea-mucosal disease infec- 
tion in pregnant cattle. J.A.V.M.A 156: 1443-1450.

Nomina Anatomica Vetrineria, fifth edition (2005): prepared by the Inter-national Committee on Veterinary Gross Anatomical Nomenclature (I. C. V. G. A. N). Published by the editorial committee, Hannover, Columbia, Gent, Sapporo.

Pearson, A.A. (1980): The development of the eyelids. Part I. External features. J of Anat. 130: 33-42.

Prasad, R and Sinha, R.D. (1979): Histological and certain histochemical studies on sebaceous glands and other modification in the eyelids of the Indian buffaloe (Bulbalus bubalis). Indian. Vet. J. 56: 672-674.

Schummer, A.; Wilkens, H.; Vollmerhaus; and Habermehl, K. $\mathrm{H}$. (1981): Skin and cutaneous organs of the domestic mammals. Vol (III), Verlag, Paul Perry, Berlin. Hamburg.

Sevel, D. (1988): A reappraisal of the development of the eyelids. Eye 2: 123-129.

Tao, H., Shimizu, M., Kusu-moto1, R., Ono, K., Noji, S. and Ohuchi, $\mathrm{H}$. (2005): A dual role of FGF10 in proliferation and coordinated migration of epithelial leading edge cells during mouse eyelid development. Development 132: 3217-3230

Teraishi, T. and Yoshioka, M. (2001): Electronmicroscopic and immunohistochemical studies of eyelid reopening in the mouse. Anat Embryol. 204: 114-107.

Tucker, S.M. and Linberg, J.V. (1994): Vascular anatomy of the eyelids. Ophthalmology 101:1118 1121

Williams, P.L. and Warwick, R. (1980): Gray,s Anatomy. $36^{\text {th }}$ ed. Churchill Livingstone, Edinburgh,
London, Melbourne and New York.

Wolfram-Gabel, R. Sick, H. (2002) microvasculariza-tion of the mucocutaneous junction of the eyelid in fetuses and neonates. Surg.Rdadiol.Anat. 24: 97-101

Zammaretti, P. and Zisch, A. H. (2005): Adult endothelial progenitors cells, Renewing vasculature. The international Journal of biochemistry and cell biology., 37: 493-503.

Zayed, A.E. (2004): The prenatal development of the eyelids of the one-humped camel (Camelus dromedarius). Assiut Vet. Med. J. 50: 37-52.

Zenz, R., Scheuch, H., Martin, P., Frank, C., Eferl, R., Kenner, L., Sibilia, M. and Wagner, E. F. (2003). C-Jun regulates eyelid closure and skin tumor development through EGFR signaling. Dev. Cell 4: 879-889.

Zhang, L., Wang, W., Hayashi, Y., Jester, J. V., Birk, D. E., Gao, M., Liu, C. Y., Kao, W. W., Karin, M. and Xia, Y. (2003). A role for MEK kinase 1 in TGF-beta/ activin induced epithelium movement and embryonic eyelid closure. EMBO J. 22: 4443-445 
Figure (1):

Panel (A) Photograph of $29 \mathrm{~cm}$ CVRL (139 days) fetus showing the fused eyelids and the emerged hairs of the eyebrow (arrows).

Panel (B) Stereomicroscopic photograph of a posterior view of the same age fetus shows the posterior bulging of fusion zone (arrows) X4.

Panel (C) Posterior view of the eyelids in $60 \mathrm{~cm}$ CVRL (209 days) fetus showing opened palpebral fissure (arrow heads). The inset is a stereomicroscopic photograph of the posterior part of eyelid margin showing the small papillae of the openings of tarsal glands (arrows) X10.

Panel (D) Stereomicroscopic photograph of the anterior surface of the lower eyelid in 80 $\mathrm{cm}$ CVRL (254 days) showing the rostrally and ventrally directed cilia (arrow heads) and the small papillae of the openings of tarsal glands (arrows) X4.

Panel (E) H \& E paraffin section of developing eyelids in $6.2 \mathrm{~cm}$ CVRL (56 days) showing the fusion zone (arrow) between upper (1) and lower (2) eyelids, conjunctival sac (star) cornea (C) and lens (L). The inset is a higher magnification of the fusion zone.

Panel (F) H \& E paraffin section of developing eyelids in $8.5 \mathrm{~cm}$ CVRL (67 days) showing the primordia of 3 rd eyelid (3), upper eyelid (1), cornea (C) and lens (L). 

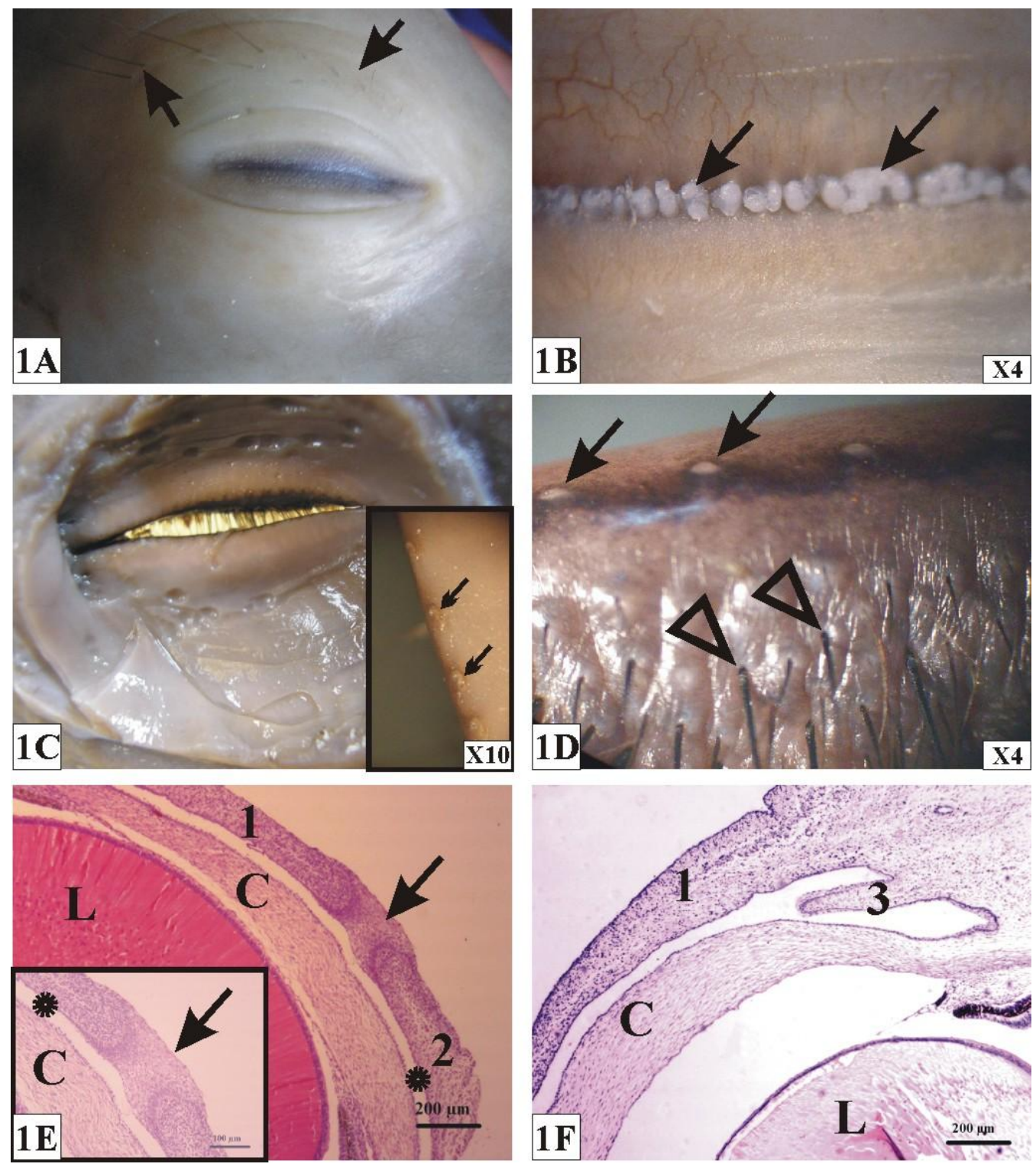
Figure (2):

panel (A) H \& E paraffin section of developing eyelids in $10.5 \mathrm{~cm}$ CVRL (76 days) buffalo fetus showing epidermis (Ep), the primordia of orbicularis oculi muscles (large arrows) and primordia of hair follicles (hair bud or plug, small arrow). The inset is a higher magnification of the primordia of orbicularis oculi muscles.

Panel (B) Higher magnification of the developing hair plugs (arrow).

Panel (C) H \& E paraffin section of developing eyelids in $13 \mathrm{~cm}$ CVRL (87 days) buffalo fetus showing developing epidermis (Ep), developing conjunctiva (Co), cornea (C), different stages of hair plugs (large and small arrows) and the initiation of tarsal glands (double head arrow).

Panel (D) H \& E paraffin section of developing eyelids in $15 \mathrm{~cm} \mathrm{CVRL} \mathrm{(96} \mathrm{days)} \mathrm{buffalo}$ fetus showing different stages of hair plugs ( $\mathrm{Hf}$ ), developing blood vessels (arrow), epidermis (Ep). The inset is a higher magnification of the developing vessels.

Panel (E) H \& E paraffin section of developing eyelids in $21 \mathrm{~cm}$ CVRL (121 days) buffalo fetus showing enlarged branched primordia of tarsal glands (Tg), developing sebaceous gland (large arrow), developing sweat gland (small arrow), epidermis (Ep). The inset is a higher magnification of the tarsal gland primordia.

Panel (F) is micro-vasculatures photograph (sagittal section) of eyelids in $19 \mathrm{~cm} \mathrm{CVRL}$ (114 days) buffalo fetus showing the main palpebral vessels (large arrows), cutaneous vessels (small arrows), main vessels of conjunctiva (double head arrows) and the initiation of deep reticular vascular networks of eyelids skin (arrow heads) $X 2$.

Panel (G) Higher magnification of panel F. X 4. 

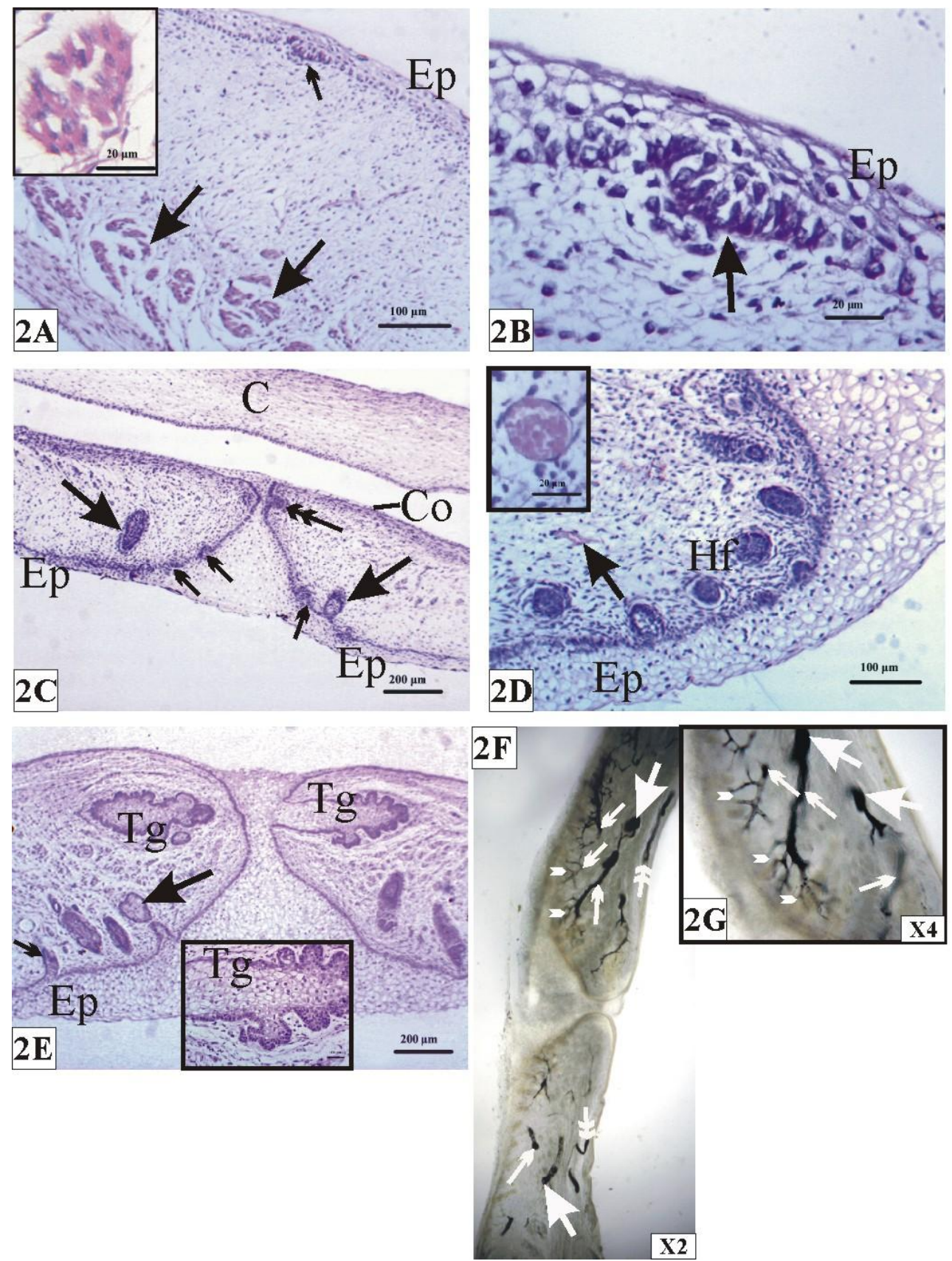


\section{Figure (3):}

panel (A) H \& E paraffin section of developing eyelids in $23 \mathrm{~cm}$ CVRL (125 days) fetus showing hair follicles (arrow heads), enlarged sweat gland (large arrow) sebaceous glands (small arrows), epidermis (Ep) conjunctiva (Co), tarsal gland ( $\mathrm{Tg}$ ), and cornea (C).

Panel (B) Higher magnification of panel (A) showing hair follicle (large arrow) the deeply embedded sweat gland (double head arrow), epidermis (Ep) and dermis (De).

Panel (C) Horizontal micro-vasculatures photograph of eyelids in $23 \mathrm{~cm} \mathrm{CVRL} \mathrm{(126}$ days) buffalo fetus shows palpebral fissure (Pf), large palpebral artery (large arrow), main veins (small arrow), branches of both vessels toward the fissure (arrow heads).

$X 2$.

Panel (D) Higher magnification of panel (C) X 10.

Panel (E) Higher magnification of panel (C) showing the venous loops (double head arrows) $\mathrm{X} 10$.

Panel (F) H \& E paraffin section of developing eyelids in $38 \mathrm{~cm}$ CVRL (159 days) buffalo fetus showing hair follicle with clear hair canal (small arrow), sebaceous gland (arrow heads), bundles of orbicularis oculi muscles (large arrows), blood vessels (double head arrows) and conjunctiva (Co). The inset is a higher magnification showing the beginning of keratinization (small arrow), hair follicle (large arrow) and enlarged sweat gland (double head arrow).

Panel (G) H \& E paraffin section of developing eyelids in $50 \mathrm{~cm}$ CVRL (186 days) buffalo fetus showing the splitting of the eyelid fusion zone (large arrow), tarsal gland with patent duct (Tg), hair shaft protruded through epidermis (arrow heads), conjunctiva (Co) and cornea $(\mathrm{C})$. The inset is a higher magnification showing melanin pigment of the epidermis (arrows). 


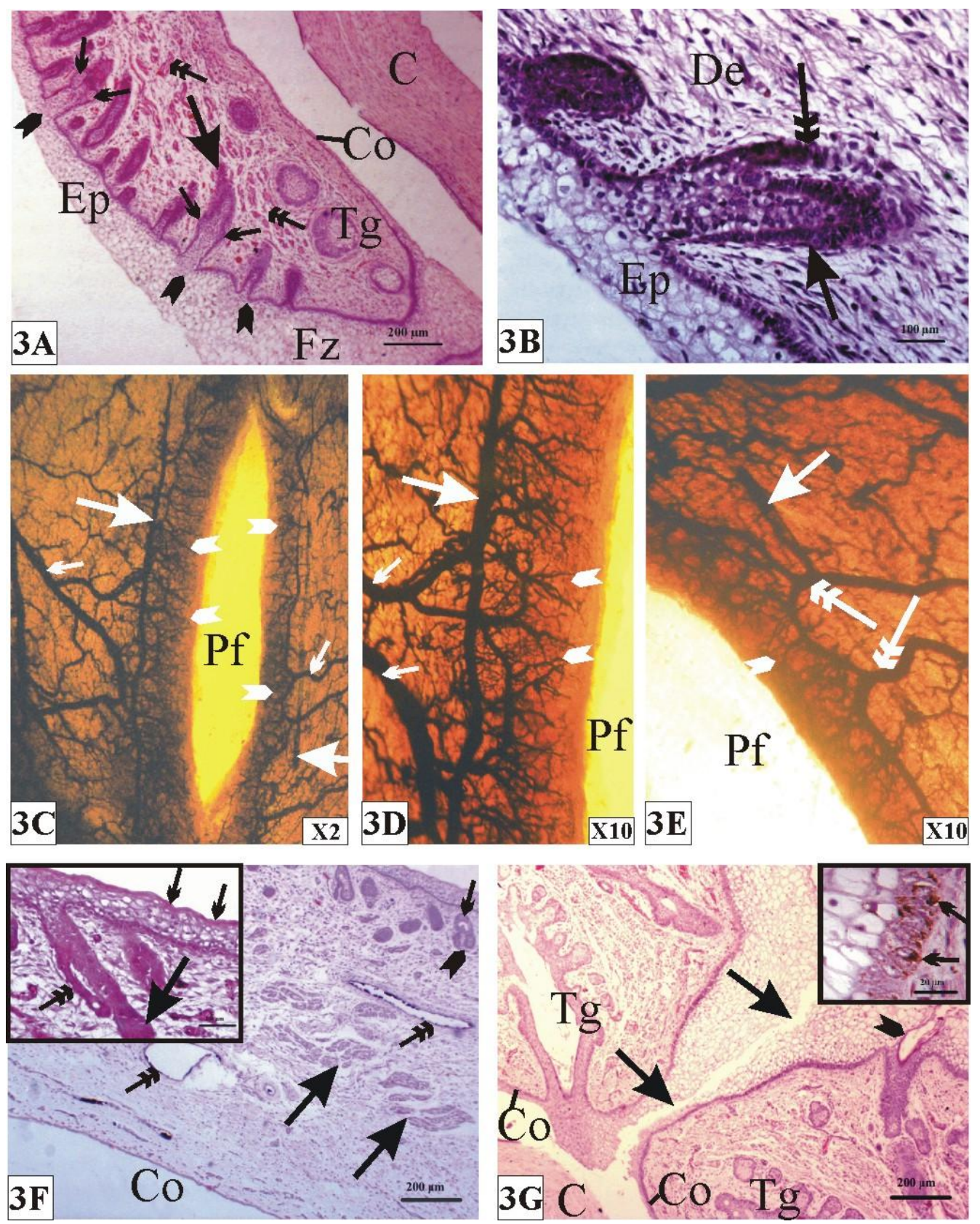


Figure (4):

panel (A) Sagittal micro-vasculatures photograph of eyelids in $46 \mathrm{~cm} \mathrm{CVRL} \mathrm{(177} \mathrm{days)}$ buffalo fetus shows main palpebral vessels (large arrow), cutaneous vessels (double head arrow), deep reticular vascular plexus (small arrow) and initiation of superficial reticular vascular networks (arrow heads) X10.

Panel (B) Sagittal micro-vasculatures photograph of the conjunctiva of the same age showing the main vessels of conjunctiva (large arrows) and the initiation of sub-mucosal vascular networks (small arrows) X10.

Panel (C) H \& E paraffin section of eyelids in $60 \mathrm{~cm}$ CVRL (209 days) buffalo fetus showing tarsal gland duct (Td), tarsal gland opening (To), keratinized skin with melanin pigment (Sk), conjunctiva (Co) and bundles of orbicularis oculi muscles (Or).

Panel (D) H \& E paraffin section of eyelids of $92 \mathrm{~cm}$ CVRL (281 days) buffalo fetus showing skin (Sk), sebaceous gland (Se) and sweat glands (arrows). The inset is a trichrome stain of the tactile hair showing the blood sinuses (stars), trabeculae of sinuses (arrows heads) and collagenous fibrous capsule (arrows).

Panel (E) H \& E paraffin section of eyelids in $103 \mathrm{~cm}$ CVRL (105 days) buffalo fetus showing thick epidermis of the free margin of the eyelid (large arrow), orbicularis oculi muscles (Or), sebaceous glands (Se), tarsal gland ( $\mathrm{Tg}$ ), sweat gland (small arrows) and hair follicle (Hf).

Panel (F) H \& E paraffin section of eyelids in $92 \mathrm{~cm} \mathrm{CVRL} \mathrm{(281} \mathrm{days)} \mathrm{buffalo} \mathrm{fetus}$ showing tarsal gland and duct ( $\mathrm{Tg}$ and $\mathrm{Td}$ ), skin (Sk) and conjunctiva (Co). The inset is a higher magnification of the initial part of conjunctiva (arrow) 

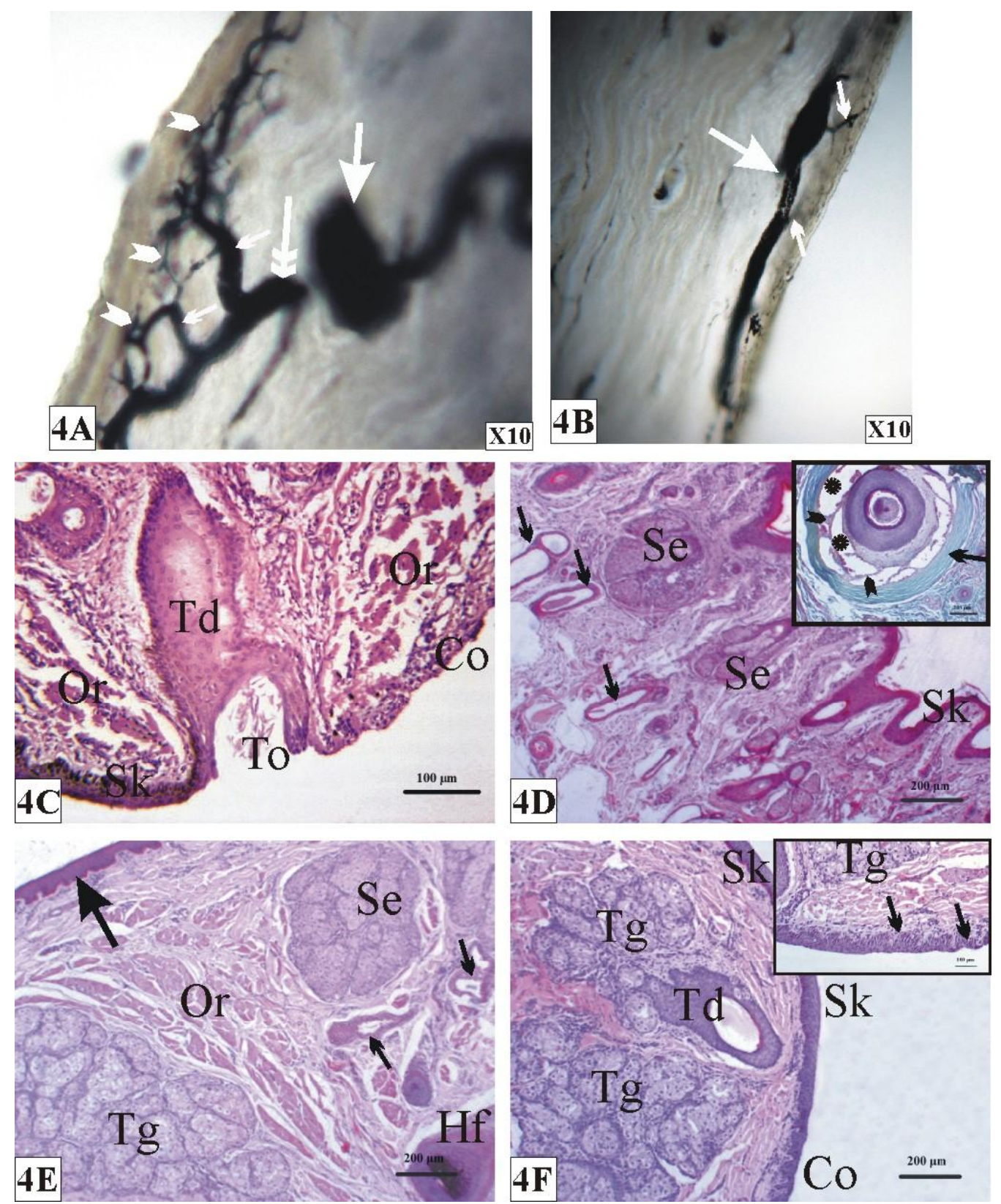


\section{Figure (5):}

panel (A) Trichrome stain of eyelid in $103 \mathrm{~cm}$ CVRL (105 days) buffalo fetus, showing thick collagenous tarsal plate (Tp), tarsal gland (Tg), tarsal gland duct (Td), orbicularis oculi muscles (arrows), and conjunctiva (Co).

Panel (B) PAS stain of the transitional epithelium of the conjunctiva (Co), showing the positively stained goblet cells (arrows).

Panel (C) Micro-vasculatures of eyelid in $80 \mathrm{~cm}$ CVRL (254 days) buffalo fetus, showing main palpebral vessel (large arrow), conjunctival vasculatures (small arrows), skin vasculatures (arrow heads), deep embedded cilia (double head arrows) and mucocutaneous junction (black arrow) $\times 2$.

Panel (D) Higher magnification of eyelid skin micro-vasculatures, showing deep reticular networks (large arrows), superficial reticular networks (small arrows) and papillary networks (arrow heads) X 10.

Panel (E) Higher magnification of conjunctiva micro-vasculatures, showing main vessels (large arrow), submucosal networks (small arrows) and mucosal networks (arrow heads) X 10 .

Panel (F) Perifollicular vascular networks (arrows) surrounding hair follicle (arrow head) $\mathrm{X} 10$. 

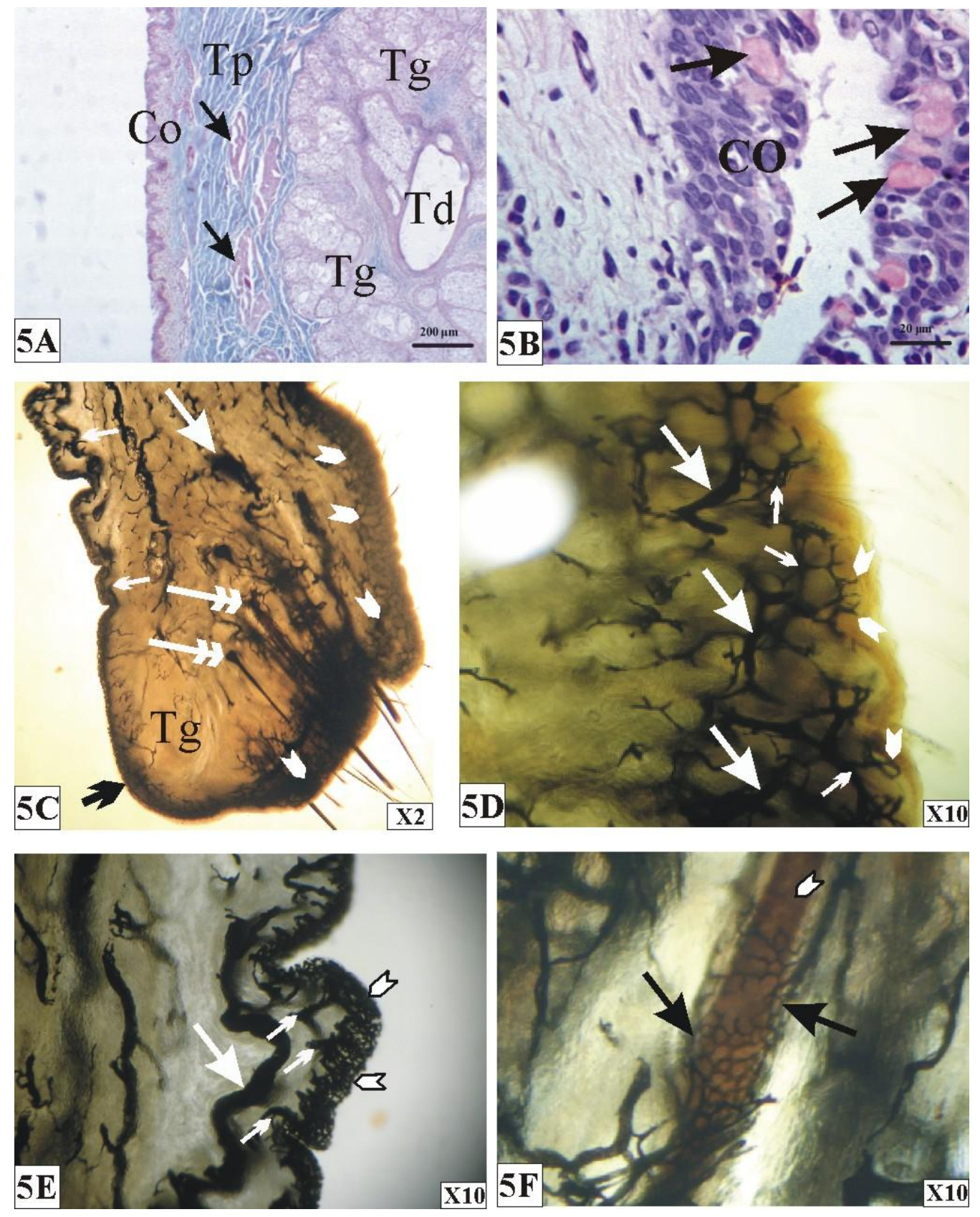